\title{
Beyond Pain Relief: Is Opioids Use Safe in Clostridium difficile Infection?
}

\author{
Hassan Al Moussawi ${ }^{\mathrm{a}, \mathrm{c}}$, Mira Alsheikh ${ }^{\mathrm{a}}$, Khalil Kamar ${ }^{\mathrm{b}}$, Zeinab Awada ${ }^{\mathrm{b}}$, \\ Jeff Hosry ${ }^{\mathrm{b}}$, Liliane Deeb ${ }^{\mathrm{a}}$
}

\begin{abstract}
Background: Clostridium difficile infection (CDI) is a common condition in hospitalized patients. In the USA, there has been an alarming rise in the use of opioids for analgesia during hospitalization. Due to their antiperistalsis effect, opioids can increase absorption of bacterial toxins. Our study aimed to highlight any correlation between opioids use in CDI and morbidity, mortality, and duration of hospitalization.
\end{abstract}

Methods: A retrospective study was performed, and data were collected from 321 hospitalized patients with CDI. The dosage of opioids received in the first 4 days following diagnosis was calculated. Patients were divided into two groups (control group vs. opioid group). Reassessment of severity of disease on day 4 was performed. Complications, hospital mortality, readmissions for CDI within 3 months, length of stay, and disposition at discharge were compared.

Results: The opioid arm consisted of 169 patients, and 152 patients served as controls. On day 4, the number of patients with severe disease was significantly higher in the opioid group versus controls (78 $(46.1 \%)$ vs. 37 (24\%), respectively, $\mathrm{P}<0.01)$, and complications including ileus, high white blood cell count, and need for vasopressors were significantly higher in the opioid group $(27.8 \%$ versus $16.4 \%$, $\mathrm{P}=0.01)$. Control group patients were more likely to be discharged home $(47 \%$ vs. $33 \%, \mathrm{P}=0.04)$, while opioid group required predominantly long-term facilities care after discharge.

Conclusion: Opioid usage for analgesia in CDI increases the risk for severe disease, complications, longer hospitalization, readmission rates, hospital mortality and discharge to a long-term facility.

Keywords: Opioids; Clostridium difficile; Severity

Manuscript submitted August 18, 2021, accepted September 23, 2021

Published online October 14, 2021

aDepartment of Gastroenterology, Staten Island University Hospital, Northwell Health, Staten Island, NY, USA

bDepartment of Medicine, Staten Island University Hospital, Northwell Health, Staten Island, NY, USA

${ }^{\mathrm{c} C}$ Corresponding Author: Hassan Al Moussawi, Department of Gastroenterology, Staten Island University Hospital, Northwell Health, Staten Island, NY,

USA.Email: mousawi_hassan@hotmail.com

doi: https://doi.org/10.14740/gr1453

\section{Introduction}

Clostridium difficile infection (CDI) is the most common cause of healthcare-associated infections in the USA and was the leading cause of enterocolitis-associated death in 2007 [1, 2]. With the appearance of new strain BI, NAP1, or ribotype 027, CDI was noted to be more common and severe. It developed resistance to medical therapy and was associated with higher recurrence rate. In 2011, an estimated 453,000 new cases of CDI occurred in the USA, of which 29,300 patients died [2]. Factors that increase the risk of CDI include malignancy, antibiotic usage, increased number of comorbidities, chronic kidney disease, inflammatory bowel disease, cirrhosis, gastrointestinal surgery, proton pump inhibitors (PPIs), histamine $\mathrm{H}_{2}$-receptor antagonists, and steroid use. Fortunately, affected by a decrease in healthcare-associated infections, the incidence of CDI declined (154.9 to 143.6 per 100,000 persons) between 2011 and 2017 [3]. CDI can present with mild diarrhea, severe infection with the formation of pseudomembranes, or a fulminant colitis with toxic megacolon. Mortality rates of $C$. difficile-associated diarrhea ranged between $4.2 \%$ and $6.9 \%$ in several centers in North America; however, rates increased with age [4]. Higher death rates were noted with healthcareassociated CDI as the incidence of NAP1 strain is higher in this group [2,3]. Treatment and control of CDI represent a substantial economic burden as the healthcare cost in the USA is up to $\$ 3.2$ billion [5-7]. The associated increased morbidity, mortality, and healthcare costs have made CDI an evolving public health concern [8].

On the other hand, the increased use of opiates all over the USA is alarming. CDC declared opioid usage as a national crisis with more than 191 million opioid prescriptions in 2017 and 58.7 prescriptions per 100 people. More than three-quarters of patients with CDI were noted to receive opioids for analgesia during their hospitalization in one study [9]. With this recent opioid epidemic, it is vital to recognize the interplay between narcotics and CDI.

Due to their antiperistalsis effect, opioids lead to increased contact time of the $C$. difficile bacteria with the intestinal mucosa, increased epithelial penetration, and absorption of bacterial toxins [10]. Mora et al reported that the use of a moderate to a high dose of opioids increased the risk of contracting CDI, though the effect of opioids on the course of CDI was not adequately studied. There have been only scarce reports evaluating the impact of opioids on the severity and prognosis of CDI. 
Table 1. Opioids Conversion to MME

\begin{tabular}{llll}
\hline Analgesic & Route of administration & Dose & MME \\
\hline Morphine & Parenteral & $1 \mathrm{mg}$ & $3 \mathrm{mg}$ \\
Hydromorphone & Parenteral & $1.5 \mathrm{mg}$ & $30 \mathrm{mg}$ \\
Hydromorphone & Oral & $7.5 \mathrm{mg}$ & $30 \mathrm{mg}$ \\
Fentanyl & Parenteral & $1 \mu \mathrm{g}$ & $300 \mathrm{mg}$ \\
Fentanyl & Transdermal & $100 \mu \mathrm{g}$ & $200 \mathrm{mg}$ \\
Hydrocodone & Oral & $1 \mathrm{mg}$ & $1 \mathrm{mg}$ \\
\hline
\end{tabular}

MME: morphine milligram equivalent.

Our study aimed to highlight any correlation, independent of the severity of the disease at presentation to hospital, between opioids use in CDI and outcomes such as morbidity, mortality, and hospital stay.

\section{Materials and Methods}

A longitudinal retrospective study was conducted between December 2015 and December 2017. The patients' database available on the electronic medical record McKesson system was scanned based on predefined inclusion and exclusion criteria. Subjects were identified using the ICD-9 and 10 codes. Only patients over the age of 18 , with three or more unformed stool and diagnosed with CDI by polymerase chain reaction (PCR) were included. Pregnant patients, those with other concomitant active infections, acute kidney injury due to causes other than CDI, and hospitalization for less than 4 days were excluded. Patients on chronic narcotics were also excluded. A total of 321 hospitalized patients with CDI were enrolled. Approval was obtained from the Institutional Review Board. This study was done in accordance with the ethical standards of the institutional and/or national research committee and with the 1964 Helsinki declaration and its later amendments or comparable ethical standards.

Opioid analgesics included acetaminophen/hydrocodone, fentanyl, morphine, hydromorphone, oxycodone, and oxycodone/acetaminophen. The total dosage of opioids received in the first 4 days following CDI diagnosis was calculated. The doses of different types of opioids received were converted into morphine milligram equivalents (MMEs) as shown in Table 1 [11].

Patients were divided into two groups depending on whether they received opioids or not. All gathered data were grouped in one confidential electronic database using REDCap electronic data capture tools (Vanderbilt University, Nashville, $\mathrm{TN}$ ) and handed to the principal investigator for grouping and statistical analysis. The primary outcome was the assessment of CDI severity at day 4, according to the American College of Gastroenterology CDI severity scoring system. We used the following predictive criteria of unfavorable outcomes to classify severe CDI: white blood cell (WBC) count 15,000 cells/ $\mathrm{mm}$ or serum creatinine $>1.5 \mathrm{mg} / \mathrm{dL}$. Complicated CDI included patients who developed any of the following: ileus, toxic megacolon, temperature $>38.5^{\circ} \mathrm{C}\left(101.3^{\circ} \mathrm{F}\right)$, hypotension,
Table 2. Primary and Secondary Outcomes of CDI in Control and Opioid Groups

\begin{tabular}{llll}
\hline Outcome & Opioid group & Control group & P-value \\
\hline Severe CDI & $78(46.1 \%)$ & $37(24 \%)$ & $<0.001$ \\
Complicated CDI & $47(27.8 \%)$ & $25(16.4 \%)$ & 0.01 \\
LOS (days) & $13(6-18)$ & $7(5-11)$ & $<0.001$ \\
Hospital mortality & $29(17 \%)$ & $13(9 \%)$ & 0.022 \\
Home disposition & $57(33 \%)$ & $71(47 \%)$ & 0.04 \\
\hline
\end{tabular}

CDI: Clostridium difficile infection; LOS: length of stay.

the need for vasopressors, WBC count $>35,000$ or $<2,000$, lactate $>2.2$, altered mental status, and the need for surgery. Complications, hospital mortality, readmission for CDI within 3 months, length of stay (LOS), peak WBC count, peak creatinine, and disposition at discharge were compared between the two study arms and served as secondary outcomes.

\section{Statistical analysis}

Univariate and multivariate analyses were used to identify possible risk factors, LOS, and hospital outcomes in patients receiving opioids for analgesia compared to controls. Data analysis was performed using SPSS statistical analysis software. Statistical significance was set at $\mathrm{P}$-value $<0.05$ with a power of 0.8 and a 1:1 subject to control ratio. Logistic regression analysis was conducted to control for confounding factors including diabetes, hypertension, coronary artery disease, liver cirrhosis, congestive heart failure, previous history of CDI, chronic kidney disease, malignancy, or immunosuppression.

\section{Results}

A total of 321 hospitalized patients with CDI were enrolled. The opioid arm consisted of 169 patients with CDI who received opioids (52.6\%). All other 152 (47.4\%) patients served as the control group. Primary and secondary outcomes of patients in the opioid group and controls were evaluated (Table 2).

On the fourth day from diagnosis, the number of patients with severe disease was significantly higher in the opioid group versus controls (78 (46.1\%) vs. 37 (24\%), respectively, $\mathrm{P}<0.01$ ) (Fig. 1). After adjusting for the severity of infection at the time of diagnosis (i.e., whether patient had mild or severe disease), the opioid group still showed a remarkable worsening of their disease status on day 4 from diagnosis $(\mathrm{P}<$ $0.01)$ compared to controls.

Logistic regression analysis was used to identify risk factors of severe CDI at day 4. Opioid use was found to independently increase the risk of severe CDI at day 4 of admission by four-folds (odds ratio (OR): 4.089; confidence interval (CI): 2.32 - 7.18; $\mathrm{P}<0.001)$. Patients with history of immunosuppression, antibiotics, PPI usage, and advanced age were also at a higher risk of developing severe CDI at day 4 of hospitaliza- 


\section{Percentage}

60

$\mathrm{P}<0.01$

50

40

$46.1 \%$

30

20

10

0

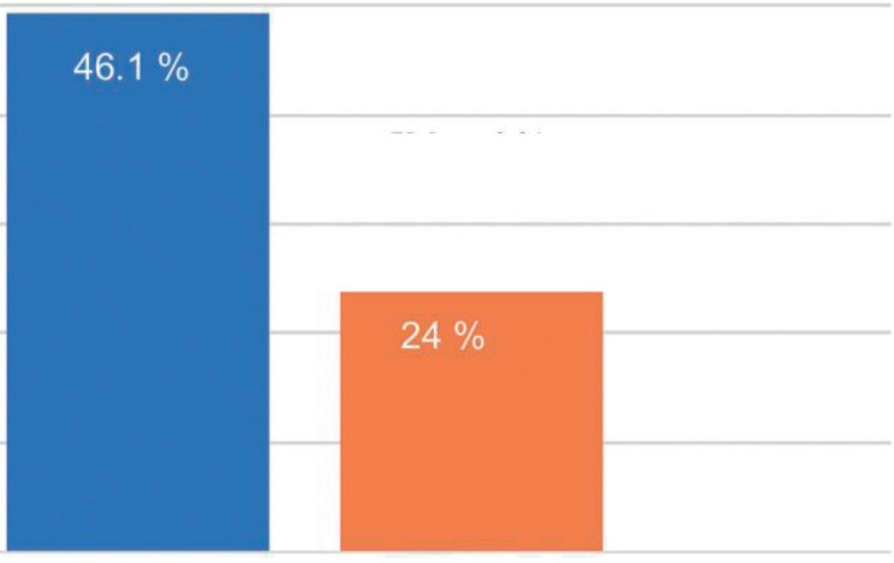

severity of the disease

- Opioid arm =Control Arm

Figure 1. Severity of CDI (percentage of patients with severe disease) on day 4 of hospitalization in patients receiving opioids for pain versus controls. CDI: Clostridium difficile infection.

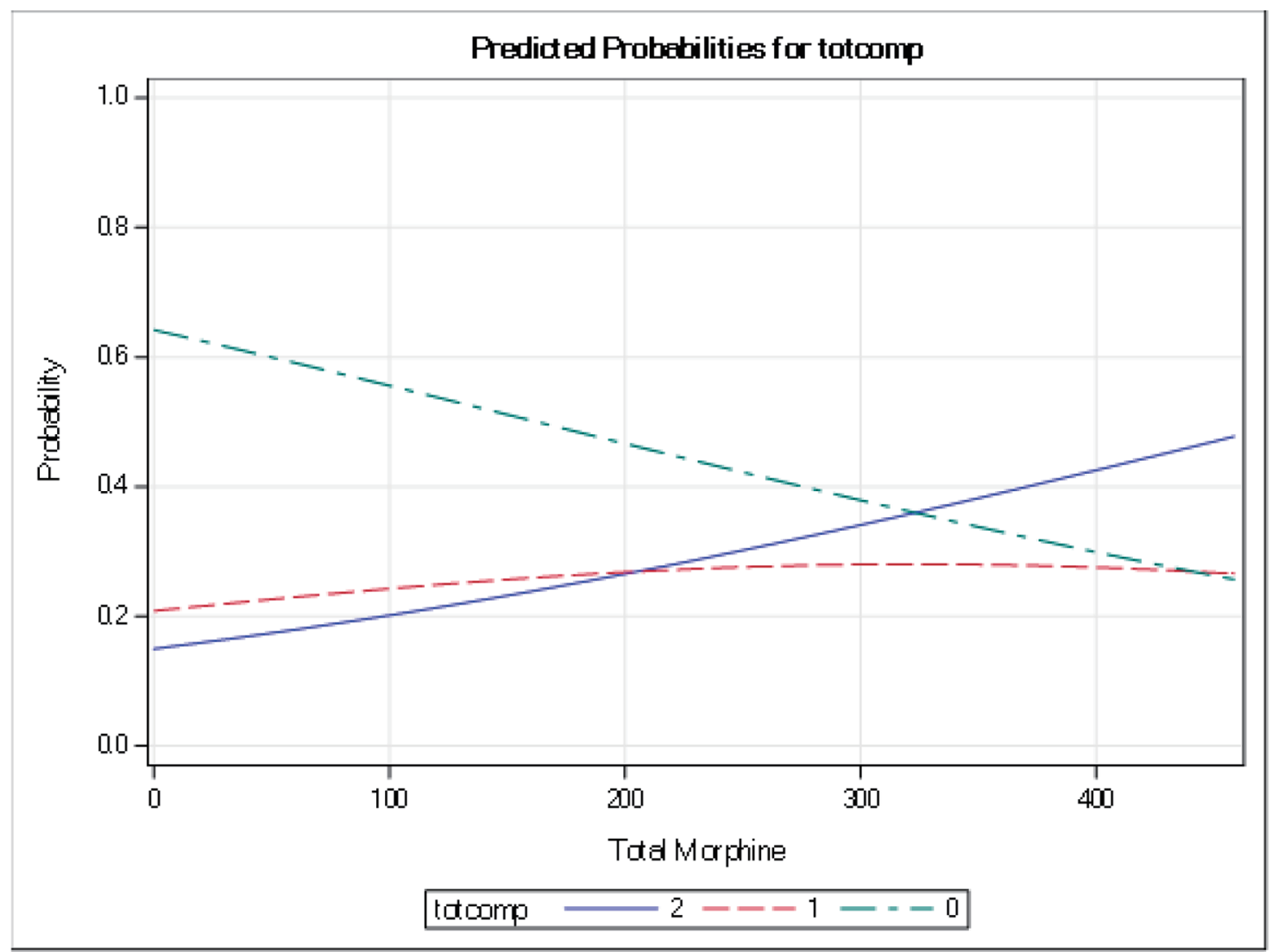

Figure 2. Predicted probability of total number of complications in patients with CDI receiving opioids for pain control. CDI: Clostridium difficile infection. 


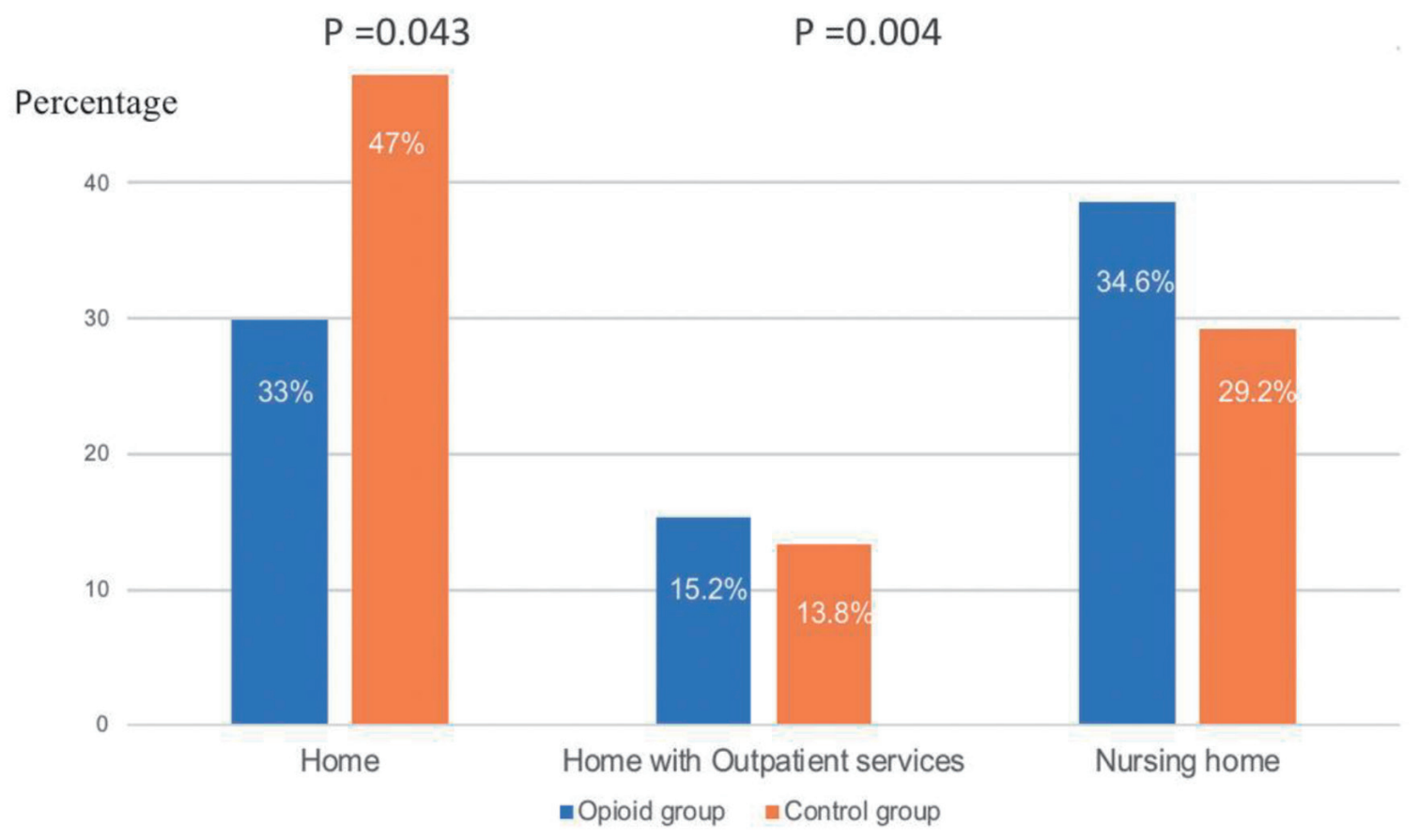

Figure 3. Disposition of patients with CDI who received opioids for pain versus controls. CDI: Clostridium difficile infection.

tion.

Complications including intestinal ileus, leukocytosis, or leucopenia (WBC count $>35,000$ or $<3,000$ ), lactic acidosis, and the need for vasopressors were significantly higher in the opioid group $(27.8 \%$ versus $16.4 \%, \mathrm{P}=0.01)$. The number of complications increased proportionally with the total dosage of morphine equivalent received (Fig. 2). Hospital mortality in the opioid group was significantly higher $(29$ (17\%) versus 13 $(9 \%), \mathrm{P}=0.022)$.

Patients in the opioid group had significantly longer LOS compared to the control group (13 days (6 - 18) versus 7 days (5 - 11), $\mathrm{P}<0.001)$. Regarding patients presenting with mild CDI, readmissions within the next 3 months were significantly higher in the opioids group compared to the controls (18 $(24 \%)$ vs. $5(8 \%), \mathrm{P}=0.01)$. Peak $\mathrm{WBC}$ and peak creatinine were higher with opioids, but not statistically significant. Control group patients were more likely to be discharged home compared to the opioid group (71 (47\%) versus 57 (33\%)). Patients in the opioid group were more likely to require extended care predominantly at long-term facilities or skilled nursing homes or required additional health services if they were to be discharged home compared to the control group (112 (67\%) vs. $81(53 \%) ; \mathrm{P}=0.004)$ (Fig. 3).

\section{Discussion}

For more than three decades, the usage of antimotility agents during the management of bacterial enterocolitis has been intensely discouraged. Intestinal motility is a biological defense mechanism against enteric infections by promoting intestinal clearance of the infecting organisms $[12,13]$. Toxins A and B play a fundamental role in CDI disease. Although the role of toxin B is incompletely understood, it is believed that both toxins cause cellular necrosis and death of the intestinal mucosal cells, increased intestinal permeability, and neutrophil infiltration of the lamina propria. These toxic effects lead to marked intestinal injury and inflammation. It has been suggested that decreased intestinal peristalsis may allow for increased contact time between organisms such as $C$. difficile, their toxins, and the mucosal epithelium. Opioids are known to decrease intestinal motility; they might also result in alteration of the gut microbiome and bacterial translocation. Clostridium was reported to be one of the pathogenic bacteria that was more abundant after morphine therapy [14]. Moreover, morphine has been shown to enhance persistent inflammation by leading to endotoxin tolerance and increased absorption of $C$. difficile bacterial toxins, thus resulting in septicemia and septic shock [15]. This explains the increased risk for severe disease and associated morbidities, as shown in this study. On the other hand, the increase in discharge to a rehabilitation facility and the need for home health services is a consequence of the increased length of hospitalization in patients in the opioids group.

An abstract by John et al published in the American Journal of Gastroenterology had a similar conclusion: more than one-third of the patients using opioids for analgesia in CDI developed progression to severe or complicated CDI [16]. The study was limited by a higher prevalence of immunosuppression and chemotherapy use in the opioid group (19.2\% vs. $12.5 \%$ and $11.8 \%$ vs. $6.3 \%$, respectively), which can adversely limit the assessment of severity.

Another abstract by Chowdhry et al [17] similarly showed an increased risk of severe CDI in patients exposed to opioids along with an extended LOS (the mean hospital stay was $63 \%$ 
Table 3. Baseline Characteristics of Patients in Opioid Group and Controls

\begin{tabular}{llll}
\hline Baseline characteristics & Opioid group & Control group & P-value \\
\hline Age & $71(58-81)$ & $76(63-82)$ & 0.115 \\
Male & $62(37 \%)$ & $64(42 \%)$ & 0.321 \\
White & $124(73 \%)$ & $121(80 \%)$ & 0.20 \\
Hospital acquired CDI & $72(43 \%)$ & $62(41 \%)$ & 0.42 \\
Antibiotic use & $58(34 \%)$ & $57(38 \%)$ & 0.553 \\
PPI use & $76(45 \%)$ & $67(44 \%)$ & 0.87 \\
H2-blocker use & $9(5 \%)$ & $12(11 \%)$ & 0.08 \\
Cirrhosis & $8(5 \%)$ & $2(1 \%)$ & 0.1 \\
Malignancy & $42(25 \%)$ & $32(21 \%)$ & 0.42 \\
Previous history of CDI & $36(21 \%)$ & $33(22 \%)$ & 0.92 \\
Nursing home resident & $35(21 \%)$ & $30(20 \%)$ & 0.82 \\
Immunosuppression & $24(14 \%)$ & $18(12 \%)$ & 0.53 \\
Diabetes mellitus & $64(38 \%)$ & $48(32 \%)$ & 0.23 \\
Chronic kidney disease & $29(17 \%)$ & $43(28 \%)$ & 0.017 \\
Congestive heart failure & $21(12 \%)$ & $31(20 \%)$ & 0.053 \\
\hline
\end{tabular}

CDI: Clostridium difficile infection; PPI: proton pomp inhibitor.

longer than controls).

Increased severity and hospital stay have detrimental effects on patients care and healthcare expenditure. However, poor control of the pain induced by CDI can be inconvenient for the patients and their families. Pain management is indicated to prevent adverse effects on patients' physical and psychological capacities. Therefore, heightened awareness is warranted to seek alternative analgesic agents or limit the use of opioids cautiously. Care providers should be aware of the adverse effects of opioids especially if a higher dose of opioids was deemed necessary for pain control in patients with CDI.

This study is limited by its single-centered, retrospective design. Moreover, Staten Island is a borough with a particularly high incidence of opioid pill abuse and misuse; hence our findings might not be reflective of other regions across the USA.

A major strength in our study compared to other studies is the adjustment of the results to baseline severity of CDI at the time of diagnosis. This solidifies our findings that worse outcomes measured 4 days through the illness were strongly attributed to the increased usage of opioids rather than the severity of disease at presentation. Baseline characteristics were homogenous as well (Table 3 ).

\section{Conclusions}

Opioid usage for analgesia in CDI increases the risk of severe disease, complications, extended hospitalizations, readmission rates, hospital mortality, and discharge to long-term facilities. Moreover, the overall worse outcomes induced by opioids could have detrimental effects on the patients care and healthcare expenditure. To conclude, the use of opioids for analgesia in $C$. difficile-infected patients should be practiced with cau- tion. Further large prospective clinical trials are needed to validate our study findings.

\section{Acknowledgments}

The completion of this research paper could not have been possible without the participation and assistance of so many people, and their contributions are sincerely appreciated and gratefully acknowledged. I would like to express my deep appreciation to all the team members that worked hard on data collection, running the statistics and writing the manuscript, with especial thanks to Dr. Deeb for her supervision and guidance.

\section{Financial Disclosure}

None to declare.

\section{Conflict of Interest}

None to declare.

\section{Informed Consent}

Not applicable.

\section{Author Contributions}

Data collection: Hassan Al Moussawi, Khalil Kamar, and Mira 
Alsheikh. Statistics: Jeff Horsey. Manuscript writing: Hassan Al Moussawi, Mira Alsheikh, and Zeinab Awada. Manuscript revision and work supervision and guidance: Liliane Deeb. All authors reviewed the results and approved the final version of the manuscript.

\section{Data Availability}

The data supporting the findings of this study are available from the corresponding author upon reasonable request.

\section{References}

1. Hall AJ, Curns AT, McDonald LC, Parashar UD, Lopman BA. The roles of Clostridium difficile and norovirus among gastroenteritis-associated deaths in the United States, 1999-2007. Clin Infect Dis. 2012;55(2):216-223.

2. Lessa FC, Winston LG, McDonald LC, Emerging Infections Program CdST. Burden of Clostridium difficile infection in the United States. N Engl J Med. 2015;372(24):2369-2370.

3. Guh AY, Mu Y, Winston LG, Johnston H, Olson D, Farley MM, Wilson LE, et al. Trends in U.S. Burden of Clostridioides difficile Infection and Outcomes. N Engl J Med. 2020;382(14):1320-1330.

4. Loo VG, Poirier L, Miller MA, Oughton M, Libman MD, Michaud S, Bourgault AM, et al. A predominantly clonal multi-institutional outbreak of Clostridium difficileassociated diarrhea with high morbidity and mortality. N Engl J Med. 2005;353(23):2442-2449.

5. Kyne L, Hamel MB, Polavaram R, Kelly CP. Health care costs and mortality associated with nosocomial diarrhea due to Clostridium difficile. Clin Infect Dis. 2002;34(3):346-353.

6. Lawrence SJ, Puzniak LA, Shadel BN, Gillespie KN, Kollef MH, Mundy LM. Clostridium difficile in the intensive care unit: epidemiology, costs, and colonization pressure. Infect Control Hosp Epidemiol. 2007;28(2):123130 .
7. O'Brien JA, Lahue BJ, Caro JJ, Davidson DM. The emerging infectious challenge of clostridium difficile-associated disease in Massachusetts hospitals: clinical and economic consequences. Infect Control Hosp Epidemiol. 2007;28(11):1219-1227.

8. Dubberke ER, Reske KA, Olsen MA, McMullen KM, Mayfield JL, McDonald LC, Fraser VJ. Evaluation of Clostridium difficile-associated disease pressure as a risk factor for $\mathrm{C}$ difficile-associated disease. Arch Intern Med. 2007;167(10):1092-1097.

9. Chowdhry Monica, Bhaty, et al. Opioid use in Clostridium difficile infection is associated with severe disease and prolonged hospitalization. 2018. p. S416.

10. Mora AL, Salazar M, Pablo-Caeiro J, Frost CP, Yadav Y, DuPont HL, Garey KW. Moderate to high use of opioid analgesics are associated with an increased risk of Clostridium difficile infection. Am J Med Sci. 2012;343(4):277-280.

11. Gammaitoni AR, Fine P, Alvarez N, McPherson ML, Bergmark S. Clinical application of opioid equianalgesic data. Clin J Pain. 2003;19(5):286-297.

12. Sprinz H. Pathogenesis of intestinal infections. Arch Pathol. 1969;87(6):556-562.

13. Kent TH, Formal SB, Labrec EH. Acute enteritis due to Salmonella typhimurium in opium-treated guinea pigs. Arch Pathol. 1966;81(6):501-508.

14. Wang F, Meng J, Zhang L, Johnson T, Chen C, Roy S. Morphine induces changes in the gut microbiome and metabolome in a morphine dependence model. Sci Rep. 2018;8(1):3596.

15. Banerjee S, Meng J, Das S, Krishnan A, Haworth J, Charboneau R, Zeng Y, et al. Morphine induced exacerbation of sepsis is mediated by tempering endotoxin tolerance through modulation of miR-146a. Sci Rep. 2013;3:1977.

16. John ES, Huss B, Schaer DA, et al. Opioids in clostridium difficile infections: decreasing pain but increasing severity? AGA abstracts. 2018. p. Sa1781.

17. Chowdhry M, Bhaty M, Huggett A, et al. Opioid use in clostridium difficile infection is associated with severe disease and prolonged hospitalization. AGA abstract. 2018. p. Sa1843. 